\title{
An essential role for PNLDC1 in piRNA 3' end trimming and male fertility in mice
}

Cell Research (2017) 27:1392-1396. doi:10.1038/cr.2017.125; published online 10 October 2017

\section{Dear Editor,}

PIWI-interacting RNAs (piRNAs) are germ cell-specific small non-coding RNAs that are essential for silencing transposable elements. Substantial efforts in the past decade have led to an understanding of how piRNAs are made. Primary piRNA biogenesis is initiated with transcription of piRNA precursors, followed by cleavage into piRNA intermediates, and finally, maturation by $3^{\prime}$ end trimming and 2'-O-methylation. Secondary piRNA biogenesis occurs through an amplification loop (ping pong pathway); the piRNA pools generated through primary processing guide MILI protein to cleave the target RNA for piRNA generation in a feed-forward loop that accelerates production of the piRNAs. Papi/Tdrkh has been implicated in processing the $3^{\prime}$ ends of piRNAs $[1,2]$, however, Papi/Tdrkh lacks a recognized domain for nuclease activity. Recently, two independent studies identified the proteins that function as piRNA $3^{\prime}$ end trimmers in silkworms-the poly(A) specific ribonuclease PARN-1 and the PARN-like ortholog PNLDC1 [3, 4]. In Drosophila, instead of a PARN-like trimmer [5], a nuclease known as Nibbler that also shortens the length of piRNAs from their $3^{\prime}$ ends is utilized $[6,7]$. The mammalian homolog of PARN is known as PNLDC1, which is a poly(A)-specific deadenylase. Here we report that deletion of Pnldc1 results in an accumulation of $3^{\prime}$ untrimmed piRNA intermediates of 30 40 nt and robust expression of LINE1, which is the non-long terminal repeat (LTR) type of retrotransposons in the mouse genome. These 3' extended pre-piRNAs still associate with PIWI proteins, but are impaired for their function. We thus demonstrate that PNLDC1 is the mouse trimmer for $3^{\prime}$ end maturation in piRNA biogenesis.

To investigate the function of PNLDC1 during spermatogenesis, we first detected Pnldcl mRNA transcripts in major organ tissues and in various stages of spermatogenesis. Quantitative RT-PCR showed that Pnldc1 was specifically expressed in testis tissue (Supplementary information, Figure S1A). The Pnldc1 mRNA was present in postnatal mice from 1 week to 8 weeks old
(Supplementary information, Figure S1B). Moreover, the expression of Pnldc1 in spermatogonial stem cells (SSCs) is much higher than that in pachytene spermatocytes and round spermatids (Supplementary information, Figure S1C). To investigate the in vivo function of Pnldc1, we generated Pnldcl mutant mice using the CRISPR/Cas9 system. Two Pnldcl knockout mouse lines that displayed the same phenotypes were acquired and a line containing $7 \mathrm{bp}$ mutation was selected for this study (Figure 1A and Supplementary information, Figure S2A-S2F). Pnldc1 mutant females displayed normal fertility (Supplementary information, Table S1). However, Pnldc1 mutant males were sterile and had smaller testes with significant reduction in testis weight at 2 months of age (Figure 1B and 1C). Histological analysis revealed that adult Pnldc1 mutants exhibited meiotic arrest $(22 \% \pm 3 \%)$ and spermiogenic defects $(78 \% \pm 3 \%)$ in round and elongating spermatids, and eventually leads to azoospermia (Figure 1D and Supplementary information, Figure S3A-S3G). No spermatozoa were observed in the lumen of the cauda epididymis of Pnldc1 mutants (Supplementary information, Figure S3E-S3F). TUNEL assay revealed dramatically increased apoptosis (Supplementary information, Figure S3H-S3I). In order to define meiotic defects, we analyzed the assembly of synaptonemal complexes (SC) by immunolabeling the spermatocytes with antibodies against SYCP3 and SYCP1, two elements for lateral and central axis of the SC. The proportions of spermatocytes at the leptotene and zygotenestages were increased in Pnldc1 mutant testes (Supplementary information, Figure S4A-S4B). Meiotic recombination is initiated with the formation of DNA double-strand breaks (DSBs) at the leptotene stage, which can be visualized by a DSBs marker $\gamma \mathrm{H} 2 \mathrm{AX}$. In Pnldc1-deficient pachytene spermatocytes, although $\gamma \mathrm{H} 2 \mathrm{AX}$ decorated the sex chromatin, the immunolabeling of $\gamma \mathrm{H} 2 \mathrm{AX}$ extended onto autosomes (Figure 1E). Furthermore, electron microscopic studies showed uncondensed chromatin or abnormal retention of residual cytoplasm around the nucleus of late-stage spermatids in Pnldcl mutants (Figure 1F). These results suggest that Pnldc1 mutant mice exhibited defects in 

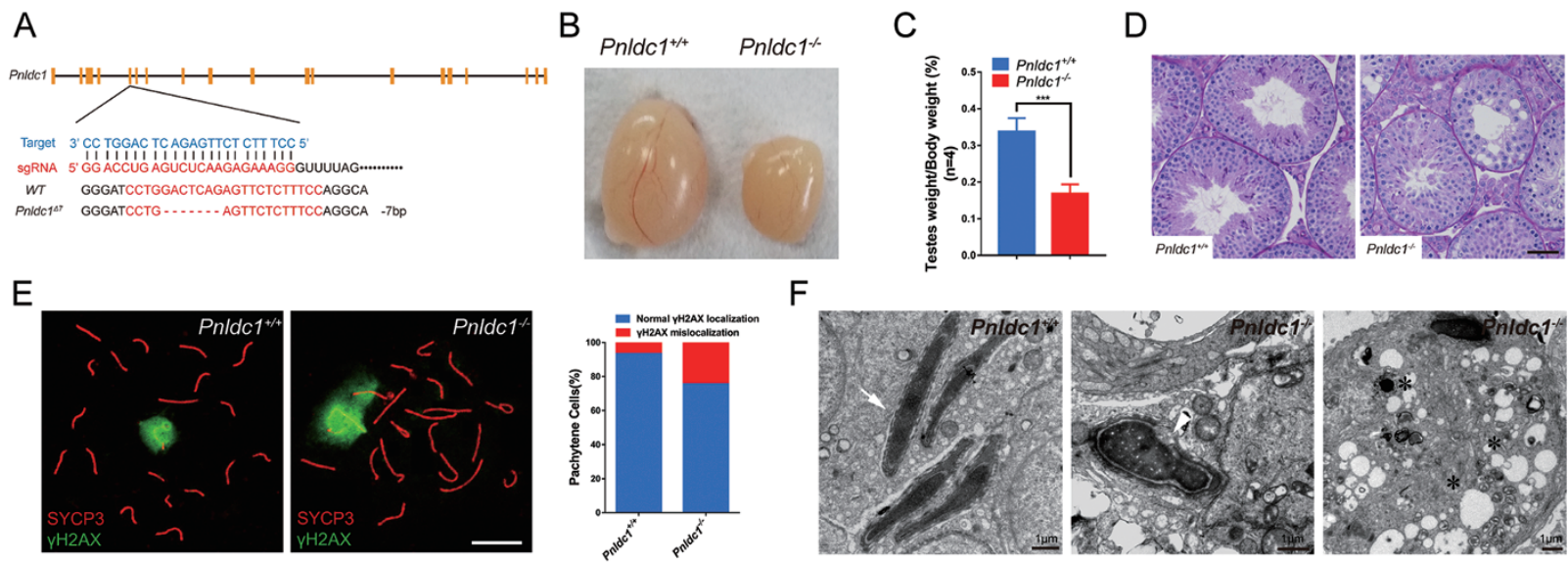

$\mathrm{F}$

G
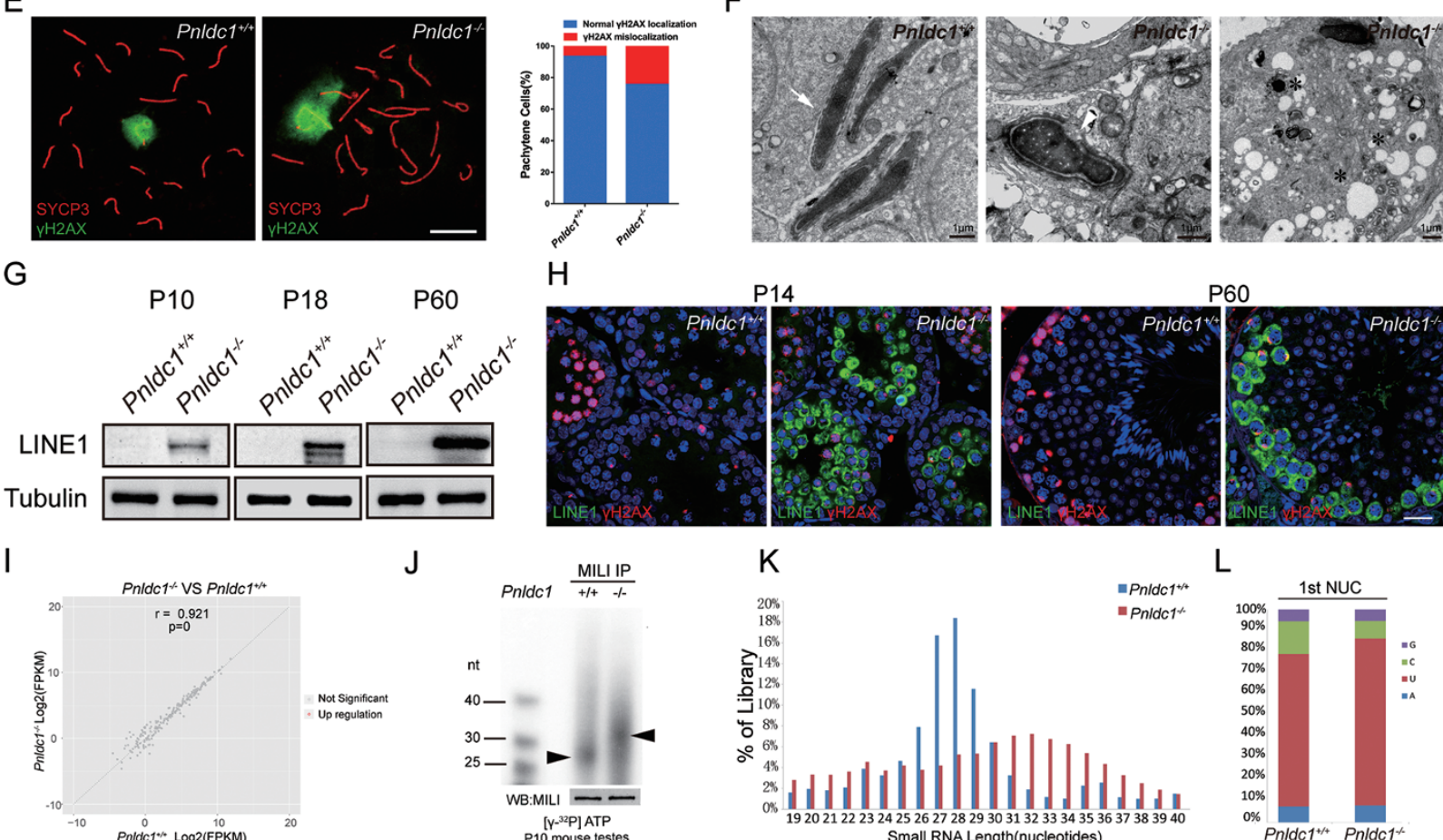

J
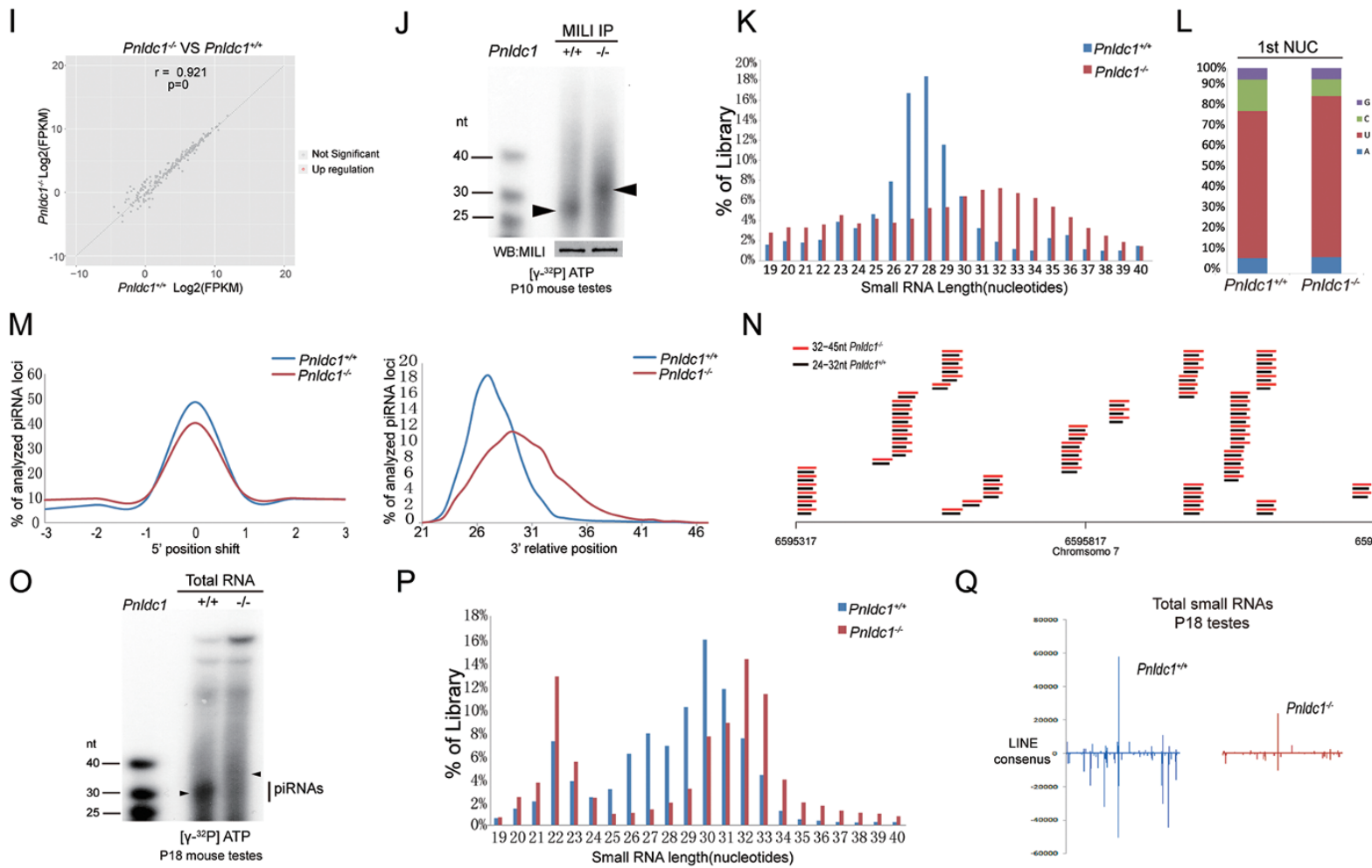

N

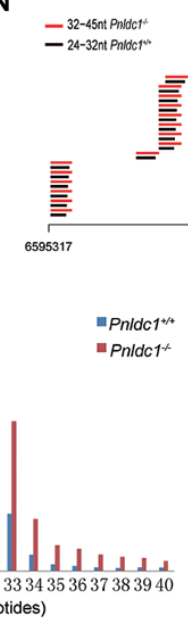

R $\quad$ MILId I IP
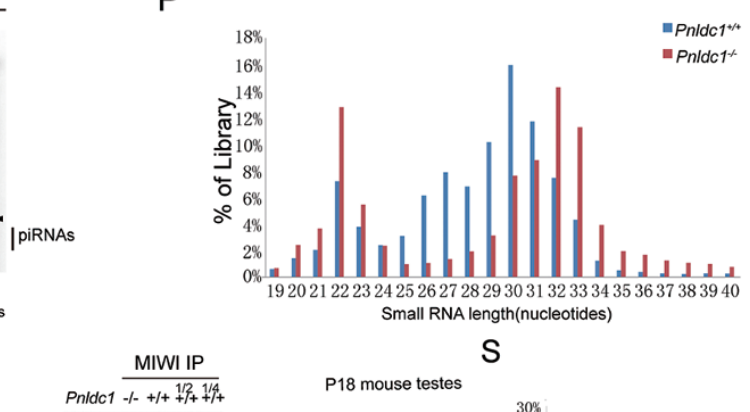

$\mathrm{S}$
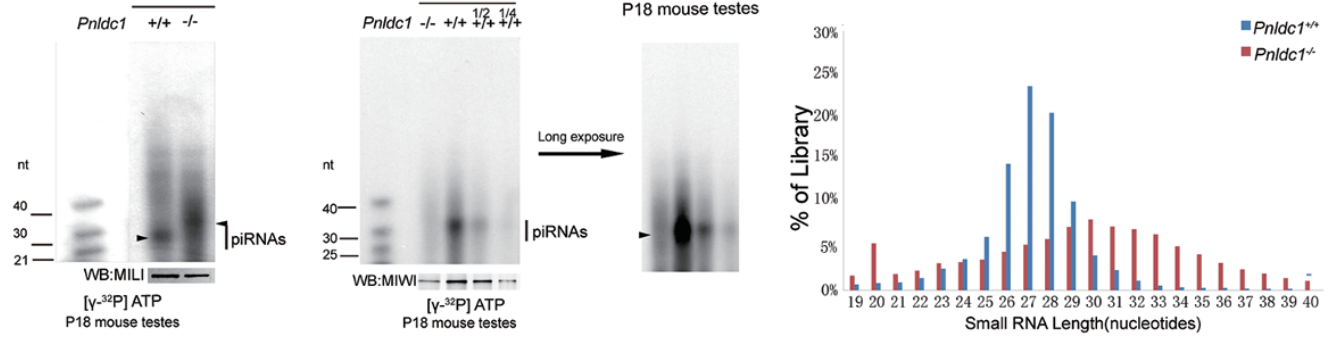

T

Q
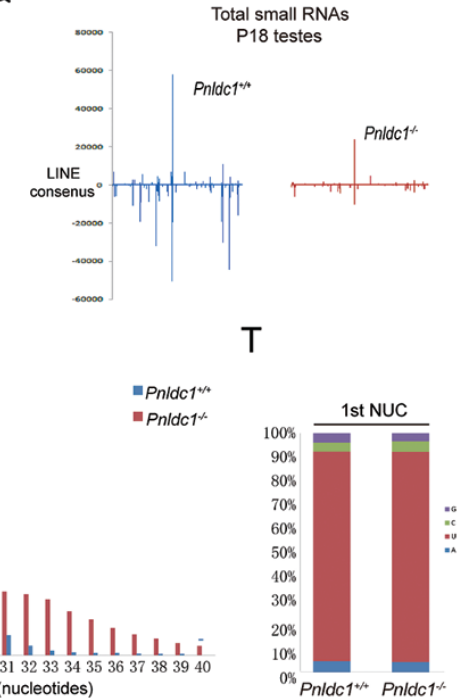

www.cell-research.com | Cell Research | SPRINGER NATURE 
meiosis and spermiogenesis.

The piRNA pathway is essential for silencing retrotransposon activity in mouse testes. In Pnldc1 mutant testes, an increase in LINE1(L1) transcripts was observed at postnatal day 18 (P18) (Supplementary information, Figure S5A).Western blot analysis showed that the abundance of L1 ORF1p, a protein product of active L1 elements, was already elevated in P10 Pnldc $1^{-/-}$testes and increased to a much higher level at P60 (Figure 1G). L1 derepression was also confirmed by immunofluorescence detection of L1 ORF1p (Figure 1H). However, the expression level of IAP, the LTR type of retrotransposons, was similar in wild type and $P n l d c 1^{-1-}$ testes (Supplementary information, Figure S5A). Thus, deletion of Pnldc1 resulted in derepression of LINE1 retrotransposons. Secondary biogenesis pathway is most active in the embryonic/perinatal male germline, and the nuclear localization of MIWI2 is dependent on piRNA loading. However, L1 was not activated in Pnldc1 mutant from P0 testes (Supplementary information, Figure S5B), and the nuclear localization of MIWI2 was observed in Pnldc1 mutant testes at P0 (Supplementary information, Figure S5C), indicating MIWI2 was normally loaded.

To address the role of PNLDC1 in piRNA biogenesis, we first deep sequenced total small RNA populations (15 45 nt) from wild-type and Pnldc1 mutant testes at P10, an early time point when L1 transposons were increased. Small RNAs with the size of 24-30 nt from 19 top pre-pachytene piRNA clusters were substantially decreased by $72.51 \%$ in Pnldc1 mutant testes compared to those in wild-type testes, whereas the abundance of 31 36 nt small RNAs derived from those clusters showed three-fold increase on average (Supplementary informa- tion, Figure S6A), indicating severe defects in piRNA biogenesis. Pre-pachytene piRNA biogenesis starts with transcription of primary piRNA precursors, and the reduced mature piRNAs in Pnldcl mutant testes could be a result of the defect in precursor processing. Therefore, we carried out deep sequencing of the transcriptome to determine the expression level of piRNA precursors. No significant changes in the expression levels of precursors were observed (Figure 1I), indicating that the transcription and stability of piRNA precursors was not affected by Pnldcl depletion. Consistent with this, RT-PCR analysis revealed a similar abundance of pre-pachytene piRNA precursor (pre-cluster 10) in wild-type and Pnldcl mutants (Supplementary information, Figure S6B). P10 testes only express MILI protein and pre-pachytene piRNAs. We next recovered MILI RNPs from P10 wild type and Pnldcl mutant testes and examined their associated piRNAs by 5 ' end labeling. Strikingly, MILI-bound piRNAs were dramatically reduced in the Pnldcl mutant (Figure 1J). Instead, the sizes of MILI-associated small RNAs were shifted to 30-40 nt (Figure 1J). Reduction of piRNAs ( $\sim 28 \mathrm{nt}$ reads) and a shift to longer lengths were also confirmed by deep sequencing of 15-45 nt MILI-associated RNAs from P10 testes (Figure 1K). Mapping of MILI-associated piRNA species to transposon consensus revealed a slight reduction in piRNA reads that mapped to sense Line1 sequences in the mutant (Supplementary information, Figure S6C). These longer RNA species (31-40 nt) in Pnldc1 mutant were strongly biased for uridine at the first position (Figure 1L), suggesting that they were processed at $5^{\prime}$ ends in the same way as mature piRNAs, but their $3^{\prime}$ ends were untrimmed. To test this hypothesis, we analyzed the perfect matches for the clus-

Figure 1 PNLDC1 is required for piRNA $3^{\prime}$ end trimming and male fertility. (A) Schematic diagram of targeting strategy by CRISPR/Cas9. (B-C) Testis size and testis weight of wild-type and Pnldc1 $1^{-/-}$mice at 8-week-old. $n=3-4,{ }^{* * *} P<0.001$. (D)

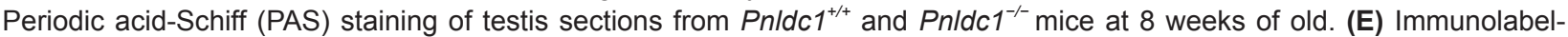
ing of SYCP3 (red) and $\mathrm{YH} 2 \mathrm{AX}$ (green) was performed on spread nuclei of pachytene spermatocytes from wild-type and $P n l d c 1^{-/-}$testes, and the quantification of $\mathrm{yH} 2 \mathrm{AX}$ mislocalization $\left(P n l d c 1^{+/+}, n=105 ; P n l d c 1^{-/-}, n=90\right)$. Scale bar, $10 \mu \mathrm{m}$. (F) Electron micrograph analysis of wild-type and Pnldc1-deficient elongated spermatids. Highly condensed chromatin of elongated spermatids in wild-type (white arrows), uncondensed chromatin (white arrowheads) and residual cytoplasm (asterisks) in elongated spermatids from Pnldc1 mutants. Scale bar, $1 \mu \mathrm{m}$. (G) Western blot analysis for LINE1 (L1ORF1p) protein levels in P10, P18 and P60 testes. $n=3-4$. (H) Immunofluorescence detection of L1ORF1p from P14 and P60 testes. (I) Relative abundance of 233 piRNA generating long single-strand transcripts in $\mathrm{P} 10 \mathrm{Pnldc1^{-/- }}$ testes compared with $\mathrm{Pnldc1^{+/+ }}$ testes by

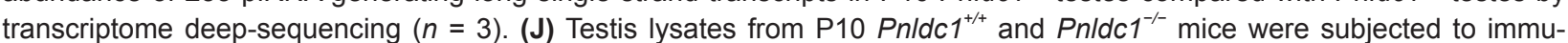
noprecipitation with anti-MILI antibody, followed by detection of MILI-associated RNAs through $5^{\prime}$ labeling. $n=10-11$. (K-L) Length distribution $(\mathbf{K})$ and the nucleotide preference at the first position (L) of MILI-associated RNAs from P10 mice. (M) The $5^{\prime}$ and $3^{\prime}$ variation analysis of piRNAs derived from the top 19 pre-pachytene piRNA clusters in P10 Pnldc1 ${ }^{+/+}$and $P n l d c 1^{-/-}$

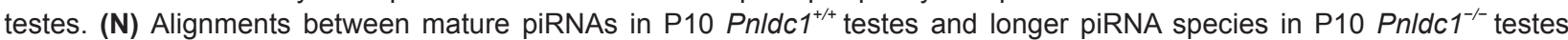
within a 1-kb window of piRNA cluster on chromosome 7. Mature piRNAs showed in black, longer piRNA species showed in red. (O) $5^{\prime}$ labeling of total small RNAs in $\mathrm{P} 18 P n l d c 1^{+/+}$and $P n l d c 1^{-/-}$testes. (P-Q) Length distribution (P) and reads mapped

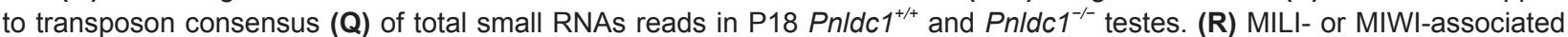
RNAs were examined by immunoprecipitation and 5' labeling. $n=3-4$. (S-T) Length distribution (S), and the nucleotide composition at the first position $(\mathrm{T})$ of MILI-associated RNAs from P18 mice. 
ter-derived MILI-associated piRNAs of 24-32 nt from wild-type testes in the cluster-derived 32-45 nt small RNA library of Pnldcl mutant. Those perfect matches were determined under the rules that they share precisely aligned 5' ends with MILI-bound piRNAs from Pnldc1 mutant. Our search revealed perfect matches for $39 \%$ of MILI-bound piRNAs of 24-45 nt reads that derived from the top 19 postnatal pre-pachytene piRNA clusters in Pnldcl mutant (statistically analyzed in Figure 1M, exemplified in Figure 1N). These results strongly suggest that the longer RNA species in Pnldc1 mutants correspond to piRNA intermediates before $3^{\prime}$ end trimming.

Pachytene piRNAs are a distinct class of piRNAs that emerge in mouse testes at P14, and their biogenesis is mainly dependent on primary pathway $[8,9]$. Similar to our findings with pre-pachytene piRNAs, the mature pachytene piRNAs were drastically reduced in Pnldcl mutant testes at P18 (Figure 1O), and this was also confirmed by deep sequencing of total small RNA populations (15-45 nt) from P18 testes (Figure 1P). Small RNAs of 24-32 nt that belong to the top 19 pachytene piRNA clusters were reduced, on average, by $88.41 \%$ (Supplementary information, Figure S7A). Mapping of total small RNAs to transposon consensus revealed a robust reduction in small RNA reads that mapped to LINE1 sequences in the mutant (Figure 1Q). Sequence annotation revealed that total small RNA reads mapped to sensestrand exonic reads in the Pnldcl mutant increased at the expense of reads mapped to the intergenic regions (Supplementary information, Figure S7B). We also examined the expression levels of piRNA precursors in P18 Pnldc1 mutant testes through deep sequencing of the transcriptome, and found no apparent changes (Supplementary information, Figure S7C). RT-PCR confirmed that the precursors of three pachytene piRNAs (piR1, piR2, piR3) were expressed at similar levels in P18 wild type and Pnldc1 mutant testes (Supplementary information, Figure S6B), while the same transcripts accumulated substantially in the testes of mice with conditional ablation of Mov10l1, a gene that was previously implicated in the primary processing of pachytene piRNAs (Supplementary information, Figure S6B) [10]. P18 testes express both MILI and MIWI proteins. We immunoprecipitated MILI and MIWI from P18 wild type and Pnldc1 mutant testes, and analyzed their associated RNAs by $5^{\prime}$ end labeling. The near absence of MILI piRNAs and a shift to longer lengths were also observed in Pnldcl mutant from P18 testes (Figure 1R). Surprisingly, MIWI-bound RNAs were almost absent in Pnldcl mutant testes (Figure 1R). Western blot and immunofluorescence analysis revealed a dramatic reduction in MIWI protein abundance (Supplementary information, Figure S8B-S8C), suggesting that the diminished MIWI piRNAs could be a direct consequence of limited MIWI protein available to bind piRNAs in Pnldcl mutants. However, unlike Miwi knockouts that uniformly arrested at the round spermatid stage, Pnldc1 mutant mice contained elongating spermatids in seminiferous tubules, showing a milder phenotype. Although MIWI protein was dramatically reduced, there was still a small amount of MIWI protein present in chromatoid body (Supplementary information, Figure S8C). After longer exposure of MIWI piRNAs from P18 mutant testes, residual untrimmed piRNAs could be detected. This suggests that residual MIWI proteins associate with untrimmed piRNAs to partially function in spermiogenesis. Reduction of MILI piRNAs $(\sim 28 \mathrm{nt}$ reads) and a shift to longer lengths were also confirmed by deep sequencing of MILI-associated RNAs from P18 testes (Figure 1S). Moreover, RNA species bound by MILI in Pnldc1 mutant testes were also strongly biased for uridine at the first position (Figure 1T). We next examined the consequences of longer pre-piRNAs/loss of mature piRNAs on the localization of piRNA pathway components including MILI, MIWI, TDRD1, TDRD6 and TDRKH. Strikingly, these proteins predominantly accumulated on one edge of the nucleus in Pnldcl-deficient pachytene spermatocytes (Supplementary information, Figure S8A). Concomitantly, mitochondria were mislocalized based on immunostaining for MT-CO1 and electron microscopy analysis (Supplementary information, Figure S8A and S8D).

In summary, here we show that Pnldc1-deficient mice exhibit spermatogenic defects in meiosis and spermiogenesis, which are accompanied by LINE1 activation. We demonstrated that PNLDC1 is required for the $3^{\prime}$ end maturation of piRNAs by processing 30-40 nt pre-piRNAs into mature piRNAs. The 5' end of untrimmed pre-piRNAs in the Pnldcl mutant perfectly match the mature piRNAs and possess the strong bias for uridine at the first position. Although these longer piRNAs can still be loaded onto MILI protein, their function is impaired. piRNAs guide PIWI protein to selectively scan the germline transcripts through sequence complementarity. The extended base-pairing posed by the longer piRNAs in the Pnldc1 mutant might decrease the efficiency of target engagement, resulting in failure to silence the L1 transponson and male sterility. However, female Pnldc1 mutants display normal fertility, which is also observed in several piRNA pathway mouse mutants with Line1 upregulation. These results indicate that female fertility is independent of piRNA-guided Line1 repression. PNLDC1 belongs to the CAF1 family, and it is the mouse homolog of $C$. elegans PARN. Therefore, PNLDC1/PARN-1 has a conserved role in $3^{\prime}$ trimming during piRNA maturation. 


\section{Acknowledgments}

This work was supported by the National Key R\&D Program of China (2016YFA0500902 and 2017YFA0103803), the National Basic Research Program of China (2015CB943003), National Natural Science Foundation of China Grant (81471502, 31771651, 31471228), Excellent Youth Foundation of Jiangsu Scientific Committee(grant BK20150047), and Innovative and Entrepreneurial Program of Jiangsu Province. We thank Jingdi Hu (Shanghai Biotechnology Corporation, Shanghai, China) for small RNA analysis.

Yue Zhang ${ }^{1, *}$, Rui Guo ${ }^{1, *}$, Yiqiang Cui ${ }^{1, *}$, Zhiping Zhu ${ }^{1,2 *}$, Yingwen Zhang ${ }^{1}$, Hao $\mathrm{Wu}^{1}$, Bo Zheng ${ }^{1}$, Qiuling Yue ${ }^{1}$, Shun Bai ${ }^{1}$, Wentao Zeng ${ }^{1}$, Xuejiang Guo ${ }^{1}$, Zuomin Zhou ${ }^{1}$, Bin Shen ${ }^{1}$, Ke Zheng ${ }^{1}$, Mingxi Liu ${ }^{1}$, Lan $\mathrm{Ye}^{1}$, Jiahao Sha ${ }^{1}$

${ }^{I}$ State Key Laboratory of Reproductive Medicine, Nanjing Medical University, Nanjing, Jiangsu, 210029, China; ${ }^{2}$ The People's Hospital of Gaochun, Nanjing, Jiangsu 210029, China
*These four authors contributed equally to this work. Correspondence: Jiahao Sha ${ }^{\mathrm{a}}$, Lan Ye ${ }^{\mathrm{b}}$, Mingxi Liu ${ }^{\mathrm{c}}$

${ }^{a}$ E-mail: shajh@njmu.edu.cn

bE-mail: lanye@njmu.edu.cn

${ }^{c}$ E-mail: mingxi.liu@njmu.edu.cn

\section{References}

1 Saxe JP, Chen M, Zhao H, et al. EMBO J 2013; 32:1869-1885.

2 Honda S, Kirino Y, Maragkakis M, et al. RNA 2013; 19:1405-1418.

3 Tang W, Tu S, Lee HC, et al. Cell 2016; 164:974-984.

4 Izumi N, Shoji K, Sakaguchi Y, et al. Cell 2016; 164:962-973.

5 Czech B, Hannon GJ. Cell 2016; 164:838-840.

6 Feltzin VL, Khaladkar M, Abe M, et al. Aging Cell 2015; 14:443452.

7 Wang H, Ma Z, Niu K, et al. Development 2016; 143:530-539.

8 Girard A, Sachidanandam R, Hannon GJ, et al. Nature 2006; 442:199-202.

9 Aravin A, Gaidatzis D, Pfeffer S, et al. Nature 2006; 442:203-207.

10 Zheng K, Wang PJ. PLoS Genet 2012; 8: 1003038.

(Supplementary information is linked to the online version of the paper on the Cell Research website.) 